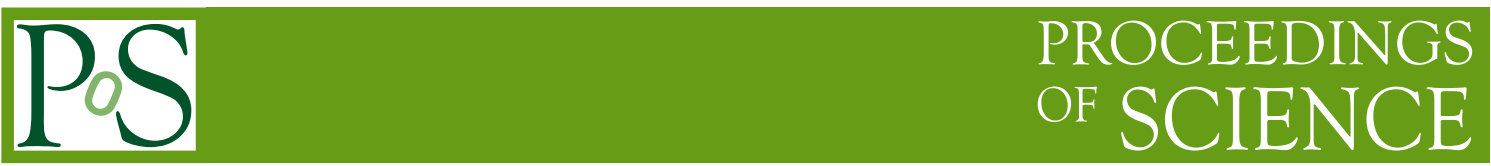

\title{
Heavy Flavour production at ATLAS and CMS
}

\author{
Adriano Di Florio* \\ on behalf of the ATLAS and CMS Collaborations \\ Universitá degli Studi di Bari Aldo Moro and I.N.F.N.-Sezione di Bari \\ E-mail: adriano.di.floriodcern.ch
}

\begin{abstract}
Studies of heavy flavour production in $p p$ collisions at the LHC both provide the means to verify the predictions of perturbative quantum chromodynamics (pQCD) and are crucial for an accurate description of background channels in Standard Model (SM) measurements and new physics searches. A wide program of studies of heavy flavour production at the LHC is performed by the ATLAS and CMS collaborations and their recent results in this field are reviewed in this paper. For the CMS collaboration, we report the measurement of differential prompt production cross sections of S-wave quarkonia in $p p$ collisions at $\sqrt{s}=13 \mathrm{TeV}$ and of production cross sections of $B_{c}^{+}\left(\rightarrow J / \psi \pi^{+}\right)$and $B^{+}\left(\rightarrow J / \psi K^{+}\right)$in $p p$ collisions at $\sqrt{s}=7 \mathrm{TeV}$. The first observation of resolved $\chi_{b 1}(3 P)$ and $\chi_{b 2}(3 P)$ peaks and the measurement of their masses through their decays to $\Upsilon(3 S) \gamma$ is also reported. For the ATLAS collaboration, recent results in measurements of pair b-quark production and the measurement of quarkonium production in $p p$ collisions at $5.02 \mathrm{TeV}$ are reviewed. Also, differential cross sections and lifetimes measurements are presented for the prompt and non-prompt production of the states $X(3872)$ and $\psi(2 S)$, in their decay mode to $J / \psi \pi^{+} \pi^{-}$in $p p$ collisions at $\sqrt{s}=8 \mathrm{TeV}$.
\end{abstract}

Sixth Annual Conference on Large Hadron Collider Physics (LHCP2018)

4-9 June 2018

Bologna, Italy

${ }^{*}$ Speaker. 


\section{Recent results in quarkonia production at ATLAS and CMS}

The study of quarkonium production is suited to understand how quarks combine into a bound state (hadron) and to test the theoretical framework of non-relativistic quantum chromodynamics (NRQCD). NRQCD is an effective theory that assumes that the quarkonium production mechanism can be factorized in two steps. In the first, an heavy $q \bar{q}$ pair is produced and the corresponding parton-level cross sections can be calculated perturbatively, presently up to NLO(Next-to-Leading Order). In the second step, the $q \bar{q}$ pairs bind into the final quarkonium states through a nonperturbative process, with transition probabilities determined by process-independent long-distance matrix elements (LDMEs). The LDMEs are currently not calculable and must be obtained by means of fits to experimental data.

\subsection{S-wave quarkonium prompt production cross sections at $\sqrt{s}=13 \mathrm{TeV}$ at CMS}

CMS [1] has recently measured differential prompt production cross sections of $J / \psi$ and $\psi(2 S)$ charmonium and $\Upsilon(n S)$ bottomonium states in $p p$ collisions at $\sqrt{s}=13.0 \mathrm{TeV}$, with data corresponding to an integrated luminosity of $2.3 \mathrm{fb}^{-1}$ for the $J / \psi$ and $2.7 \mathrm{fb}^{-1}$ for the other mesons. The five quarkonium states are reconstructed in the dimuon decay channel, for dimuon rapidity $|y|<1.2$. The double differential prompt production cross sections, times the branching fraction $(\mathscr{B})$ of the meson in two muons, are calculated, for each state, in several dimuon $p_{T}$ and $|y|$ bins, from the equation $\mathscr{B}\left(q \bar{q} \rightarrow \mu^{+} \mu^{-}\right) \times \frac{d^{2} \sigma^{q \bar{q}}}{d p_{T} d y}=\frac{\mathscr{N}^{q \bar{q}}\left(p_{T}, y\right)}{\mathscr{L} \Delta p_{t} \Delta y}\left\langle\frac{1}{\varepsilon\left(p_{T}, y\right) A\left(p_{T}, y\right)}\right\rangle$, where $\mathscr{L}$ is the integrated luminosity, $\mathscr{N}$ is the number of prompt candidates extracted in a bin of widths $\left(\Delta p_{T}, \Delta y\right)$ and $\varepsilon$ and $A$ are the efficiency and the acceptance for all the candidates in the bin. Non-prompt components for $S$-wave charmonia ( $b$ hadrons decays) are separated by using two-dimensional simultaneous fits to the pseudo-proper decay length and to the dimuon invariant mass. Directly produced quarkonia are not distinguished from feed-down decays of heavier quarkonium states (the $\psi(2 S)$ is feed-down free). The results at $\sqrt{s}=13 \mathrm{TeV}$ are compatible with NLO NRQCD predictions, shown in Figure 1 for $c \bar{c}$ states. The measurements are also compared to the $\sqrt{s}=7 \mathrm{TeV}$ results in order to check the scaling in cross sections due to the centre-of-mass energy increase. Cross sections at $13 \mathrm{TeV}$ are a factor 2-3 larger than the corresponding values at $7 \mathrm{TeV}$ and slowly increasing as a function of the $p_{T}$. An increase of this order is expected from the evolution of parton distribution functions.

\subsection{Measurement of quarkonium production in $p p$ collisions at $5.02 \mathrm{TeV}$ with the ATLAS detector}

In [2] the ATLAS collaboration has studied the modification of the production of $J / \psi, \psi(2 S)$, and $\Upsilon(n S)(n=1,2,3)$ in $p P b$ collisions with respect to their production in $p p$ collisions. Here we discuss only the results from $p p$ collisions. The $p p$ dataset used corresponds to an integrated luminosity of $25 \mathrm{pb}^{-1}$, collected in 2013 and 2015, at a $\sqrt{s}$ per nucleon pair of $5.02 \mathrm{TeV}$. The charmonium yields are separated into prompt and non-prompt sources with a two-dimensional unbinned maximum likelihood (UML) fit, performed on the dimuon invariant mass and pseudoproper life-time, in intervals of $p_{T}$ and $|y|$. The double differential cross section are measured from the yields corrected by the acceptance, integrated luminosity and reconstruction and trigger efficiencies. The results for non-prompt (prompt) $J \psi$ and $\psi(2 S)$ cross sections in $p p$ collisions 

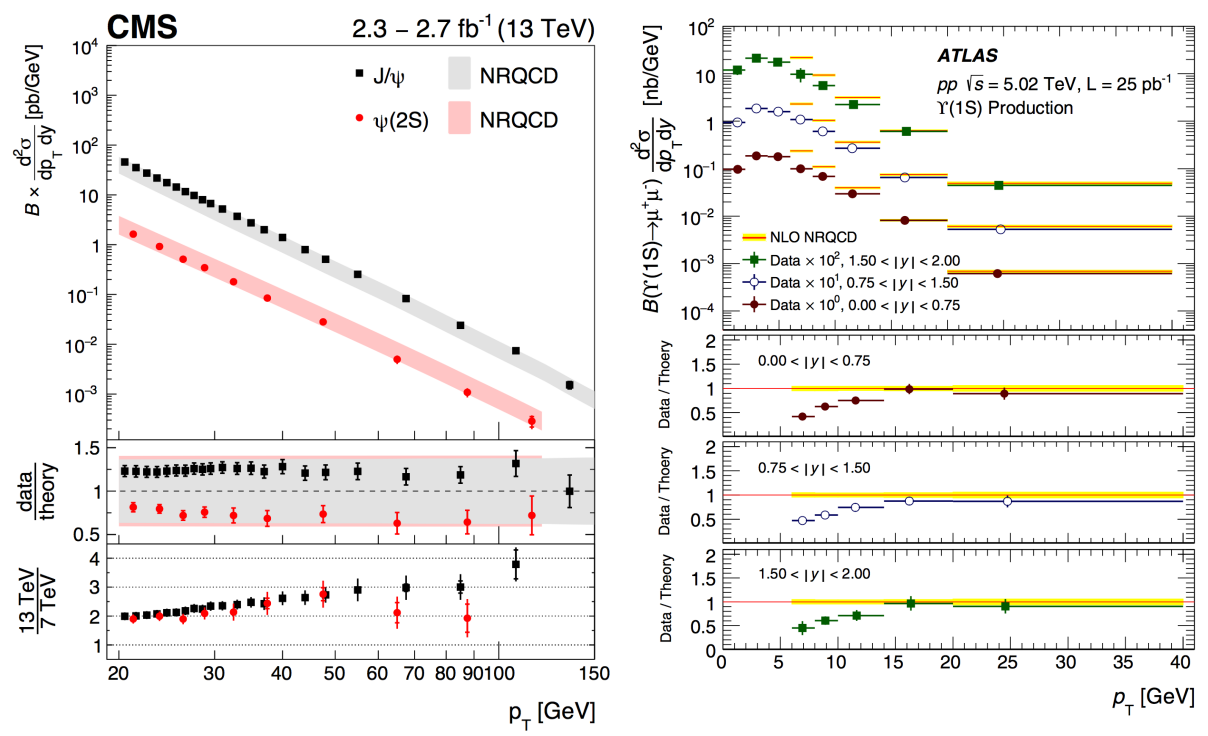

Figure 1: Left: From [1], the measured double differential cross sections times $\mathscr{B}$ of the prompt $J / \psi$ and $\psi(2 S)$ mesons compared to NLO NRQCD predictions (shaded bands). The middle panel shows the ratios of measurement to theory. The lower panel shows the ratios of cross sections measured at $13 \mathrm{TeV}$ and 7 $\mathrm{TeV}$, with their uncertainties being treated as uncorrelated. Right: from [2], the differential production cross section times dimuon $\mathscr{B}$ of $\Upsilon(1 S)$ as a function of transverse momentum $p_{T}$. The NRQCD predictions are shown with yellow error bands.

at $\sqrt{s}=5.02 \mathrm{TeV}$ agree with FONLL ${ }^{1}$ (NRQCD) predictions over the whole measured $p_{T}$ range within uncertainties, which include renormalisation, factorisation scale, $m_{c}$, LDMEs and parton distribution functions. The production cross section of $\Upsilon(n S)$ in $p p$ collisions is compared to similar NRQCD model calculations, see Figure 1 for $Y(1 S)$. As stated in [3], the LDMEs for bottomonium production are only extracted from fitting experiment data at $p_{T}>15 \mathrm{GeV}$. At lower $p_{T}$, there might be non-perturbative effects which break the NRQCD factorization and perturbation expansion. By construction, the $b \bar{b}$ NRQCD model gives a relatively good description of the measured $\Upsilon(n S)$ production cross section at $p_{T}>15 \mathrm{GeV}$, while overestimates the production cross section at lower $p_{T}$.

\section{Recent results in $b$-hadron production at ATLAS and CMS}

\subsection{Measurement of $b$-hadron pair production at ATLAS}

Small angle $b \bar{b}$ pair production is particularly sensitive to the details of heavy flavour production theoretical calculations, but remains only loosely constrained by data. The ATLAS collaboration [4] reports the measurement of $b$-hadron pair using a $11.4 \mathrm{fb}^{-1} p p$ data sample collected at $\sqrt{s}=8 \mathrm{TeV}$. One $b$-hadron is selected to decay to $J / \psi(\rightarrow \mu \mu)+X$ and the other to $\mu+X$, resulting in three $\mu$ in the final state. The signal definition includes $J / \psi$ mesons produced via feed-down from excited charmonium states as well as muons produced in semileptonic cascade decays. To probe $b$-hadron production, several differential cross sections are measured, based on the kinematics of the $J / \psi$ and the third $\mu$. To extract the $J / \psi$ mesons resulting only from the decay of a

\footnotetext{
${ }^{1}$ Fixed-to-Next-to-the-Leading Order
} 
$b$-hadron, a simultaneous fit is performed upon the distributions of $m(\mu \mu)$ and the pseudo-proper decay time $\tau$. The third muon is then selected as the highest- $p_{T}$ muon not used in the $J / \psi$ candidate reconstruction. The yield of signal events relative to background is further improved using a simultaneous fit to the transverse impact parameter significance $\left(d_{0} / \sigma_{d_{0}}\right)$ and the output of a boosted decision tree trained to separate signal muons from misreconstructed muons. Some remaining irreducible sources of background are then subtracted from the fitted yields. The experimental results are compared with Pythia 8 predictions, exploring several different options for the $g \rightarrow b \bar{b}$ splitting kernel, as this process dominates the region of particular interest, namely small angle $b$-hadron production. The measured cross sections as a function of $p_{T}(J / \psi, \mu)$ is compared to theoretical predictions, as detailed in Figure 2. The best agreement was obtained with a $p_{T}$-based scale splitting kernel. The best overall agreement is obtained with the 4-flavour Mad-Graph 5 interfaced to Pythia8, although none of the models can well-describe all the kinematic properties.

\subsection{Measurement of production cross sections of $B_{c}^{+} \rightarrow J / \psi \pi^{+}$and $B^{+} \rightarrow J / \psi K^{+}$in $p p$ collisions at $\sqrt{s}=7 \mathrm{TeV}$ at $\mathrm{CMS}$}

The $B_{c}^{+}\left(B_{c}^{-}\right)$meson is the ground state of the $\bar{b} c(b \bar{c})$ system. Similarly the $B^{+}\left(B^{-}\right)$is the ground state of $u \bar{b}(\bar{u} b)$ system and, in the SM, it is the $b$-quark meson with the largest production rate in hadron collisions. Instead the production of the $B_{c}^{ \pm}$is predicted [6] to be only $0.2 \%$ of the inclusive $b \bar{b}$ cross section, since it needs simultaneous production of two heavy quarks. The dependence of the theoretical predictions on the renormalization and factorization scales and $m_{b}$ results in theoretical uncertainties up to $40 \%$. Therefore, combined measurements of the $B^{ \pm}$and $B_{c}^{ \pm}$cross sections times their $\mathscr{B}$ can provide useful information on the production mechanism of both mesons. In [5] CMS reports the measurements of the production cross section times the $\mathscr{B}$ of $B_{c}^{+} \rightarrow J / \psi \pi^{+}$and $B^{+} \rightarrow J / \psi K^{+}$at $\sqrt{s}=7 \mathrm{TeV}$ as a function of $b$-hadron $p_{T}$ and rapidity $|y|$. The analysis is performed with the data recorded in 2011 LHC run corresponding to an integrated luminosity of $4.77 \mathrm{fb}^{-1}$. Events are selected with double muon triggers where two opposite-sign muons having a common vertex displaced from the interaction point in order to reject prompt $J / \psi$ events. Further details on the event selection can be found in Ref. [5]. The fitted yields for $B_{c}^{ \pm}$and $B^{ \pm}$are $310 \pm 36$ and $117091 \pm 347$ respectively. Figure 2 summarizes the measured differential cross sections times $\mathscr{B}$ for $B_{c}^{+}$as function of $p_{T}$ (see [5] for $|y|$ ) compared to theoretical predictions based on BCVEGPY [7]. A scale factor of 2.75 holds between theory and experiment while the shape of the measurement shows consistency with the BCVEGPY predictions, once normalised. The integrated production cross section times $\mathscr{B}\left(B_{c}^{+} \rightarrow J / \psi \pi^{+}\right)$with $p_{T}\left(B_{c}^{+}\right)>10$ $\mathrm{GeV}$ and $|y|\left(B_{c}^{+}\right)<1.5$ is measured to be $40.8 \pm 4.7$ (stat) \pm 2.8 (sys) pb. Also the differential cross section times $\mathscr{B}$ as function of $p_{T}$ and $y$ and the integrated $\sigma\left(B^{+}\right) B=5851.3 \pm 37.1$ (stat) \pm 446.4 (sys) pb with $p_{T}\left(B^{+}\right)>10 \mathrm{GeV}$ and $|y|(B+)<1.5$ are reported in [5]. All these measurement are found to be consistent with the theoretical predictions (PYTHIA, FONLL and at NLO) and with previous results from both ATLAS and CMS [5].

\section{Observation of the $\chi_{b 1}(3 P)$ and $\chi_{b 2}(3 P)$ states at CMS}

The bottomonium family plays a special role in understanding how the strong force binds quarks into hadrons within the context of QCD. The $\chi_{b}(3 P)$ is especially interesting given that 

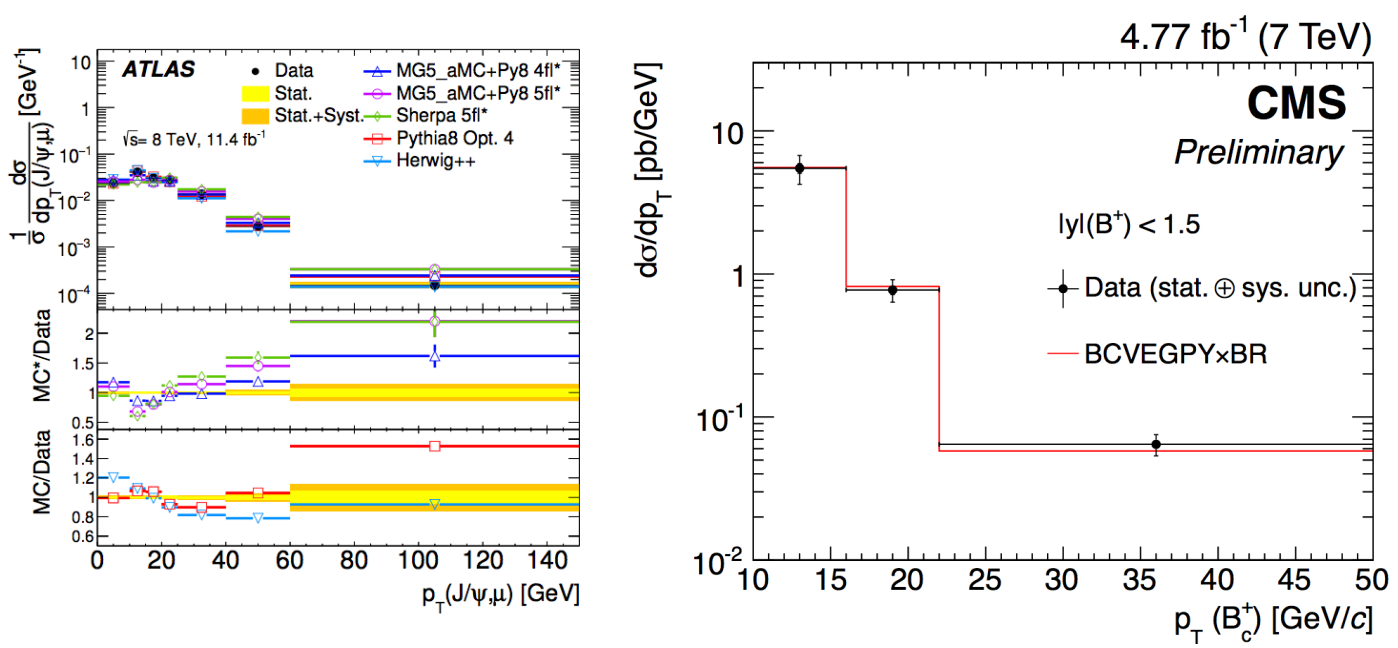

Figure 2: Left: from [4], measured normalised differential cross sections (black solid points) as a function of $T(J / \psi, \mu)$. Comparisons are made with predictions of Pythia8,Herwig++, MadGraph5 aMC@NLO+Pythia8 and Sherpa (coloured lines and bands). Right:from [5], differential production cross sections $\times \mathscr{B}$ of $d\left(B_{c}^{+}\right) / d p_{T}\left(B_{c}^{+}\right) B\left(B_{c}^{+} \rightarrow J / \psi \pi^{+}\right)$at $\sqrt{s}=7 \mathrm{TeV} p p$ collisions. Solid points with error bars are the CMS measurements and the uncertainties are statistical and systematic, respectively. The prediction of the $B_{c}^{+}$production cross section by BCVEGPY times $\mathscr{B}\left(B_{c} \rightarrow J / \psi \pi\right)=3.3 \times 10^{-03}[8]$ is scaled by 2.75 for display purposes.

the $\chi_{b j}(3 P)$ triplet states $(J=0,1,2)$ properties could be affected by the proximity of the openbeauty $(B \bar{B})$ threshold. CMS has recently reported [9] the first observation of resolved $\chi_{b 1}(3 P)$ and $\chi_{b 2}(3 P)$ states as well as the measurement of their masses. The study the $\Upsilon(3 S) \gamma$ system, with $\Upsilon(3 S) \rightarrow \mu^{+} \mu^{-}$and the photon converting into an $e^{+} e^{-}$pair, is based on $p p$ data samples collected at a $\sqrt{s}=13 \mathrm{TeV}$, from 2015 to 2017 , corresponding to an integrated luminosity of $80.0 \mathrm{fb}^{-1}$. The trigger event selection requires an opposite-sign muon pair of invariant mass within $8.5-11.5$ $\mathrm{GeV}$, with the two muons consistent with the hypothesis of originating from the same vertex and the dimuon passing $p_{T}$ and rapidity selections, with variable thresholds during the data-taking period. The selected dimuon sample contains about 2.6 million $\Upsilon(3 S)$ candidates. For an higher mass resolution at the cost of a reduced yield, if compared to calorimetric energy measurements, the analysis uses photons candidates (with $|\eta(\gamma)|<1.2$ and $p_{T}(\gamma)>500 \mathrm{MeV}$ ) converting to $e^{+} e^{-}$ pairs only in the beam pipe or in the tracker [10][11]. For a more detailed description of the event selection see Ref. [9]. The dimuon is thus combined with the converted photon to form the $\chi_{b}(3 P)$ candidate. To accurately measure the invariant mass of the $\chi_{b}(3 P)$ candidate, the photon energy scale (PES), i.e. the ratio between the reconstructed and true energy, is measured using a large sample of $\chi_{c 1} \rightarrow J / \psi \gamma \rightarrow \mu \mu \gamma$ events (collected in the same running periods as the $\Upsilon \rightarrow$ $\mu \mu$ data). The resulting distribution is parametrized with an exponential function that is then used for the event-by-event correction of the photon energy. Figure 3 shows also the final $\Upsilon(3 S) \gamma$ invariant mass distribution along with the result of an extended UML fit. The fitted number of signal events is $372 \pm 36$. The mass resolution of the low-mass peak is $2.18 \pm 0.32 \mathrm{MeV}$ while the corresponding resolutions in the $\Upsilon(1 S) \gamma$ and $\Upsilon(2 S) \gamma$ mass distributions are 7 and $15 \mathrm{MeV}$, respectively, thus justifying why only the $\Upsilon(3 S) \gamma$ distribution is used. The local significance of the double-peak structure exceeds nine standard deviations in the range $9<\Delta M<12 \mathrm{MeV}$. The $\Delta M$ 

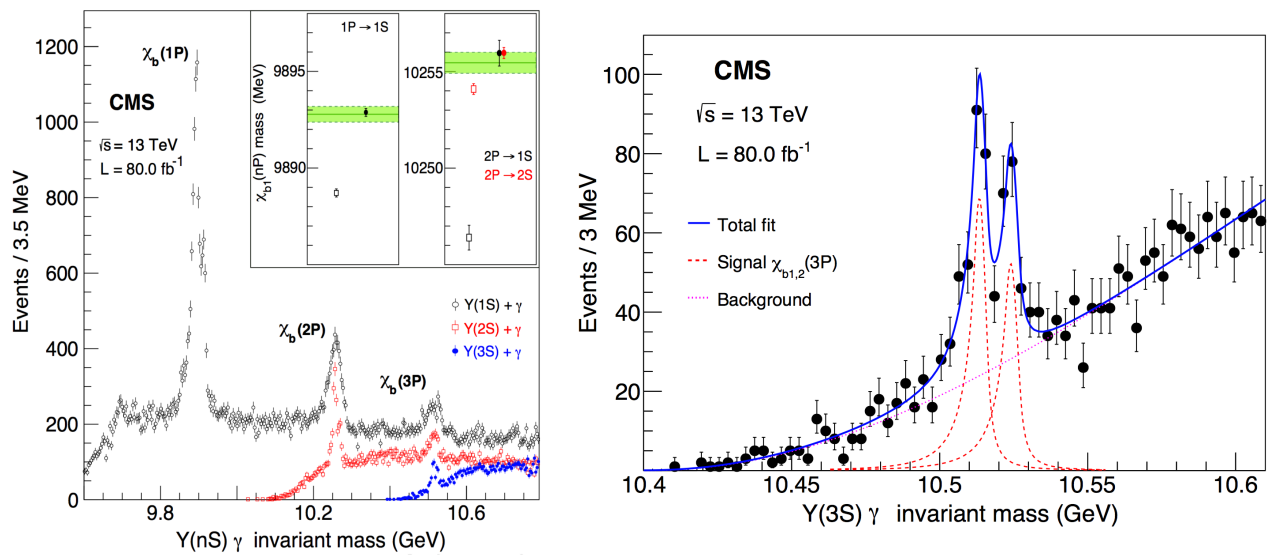

Figure 3: From [9]. Left: the invariant mass distributions of the $\chi_{b j}(3 P) \rightarrow \Upsilon(n S) \gamma$ candidates $(n=1,2,3)$, after the PES correction. The insert shows the $\chi_{b 1}(3 P)$ and $\chi_{b 1}(3 P)$ masses fitted before (open squares) and after (filled circles) the PES correction. The world-average values are shown by the horizontal bands, with dashed lines representing their total uncertainties. Right: the invariant mass distribution of the $\chi_{b j}(3 P) \rightarrow$ $\Upsilon(3 S) \gamma(J=1,2)$ candidates with the curves showing the fit results of the two signal double-sided Crystal Ball functions, the exponential background, and their sum.

and $M$ systematic uncertainties come mainly from the PES calibration function and the choice of the fitting model. The mass difference between the two states is measured to be $\Delta M=10.60 \pm$ 0.64 (stat) \pm 0.17 (syst) $\mathrm{MeV}$, while the two masses are determined to be $10513.42 \pm 0.41$ (stat) \pm 0.18 (syst) and $10524.02 \pm 0.57$ (stat) \pm 0.18 (syst) $\mathrm{MeV}$. These values can be compared to the predictions of theoretical calculations [9]. Out of $20 \Delta M$ predictions, 19 range from 8 to $18 \mathrm{MeV}$. The only exception gives $M\left(\chi_{b 2}(3 P)\right)-M\left(\chi_{b 1}(3 P)\right)=-2 \mathrm{MeV}$, the negative sign reflecting the coupling with the open-beauty threshold. The measurement reported is significantly larger than 2 $\mathrm{MeV}$ and then strongly disfavours the breaking of the conventional pattern of splittings and supports the standard mass hierarchy $(J=2$ state is heavier than the $J=1$ state).

\section{Measurements of $\psi(2 S)$ and $X(3872) \rightarrow J / \psi \pi^{+} \pi^{-}$production in $p p$ collisions at $\sqrt{s}=8 \mathrm{TeV}$ with the ATLAS detector}

The charmonium-like state $X(3872)$ was discovered by the Belle Collaboration in 2003 [12] and it was hypothesized to be a very loosely bound $D^{0} \bar{D}^{* 0}$ molecule. However, its nature is still undefined, because of non univocal results from both theoretical calculations and experimental observations. Measuring prompt and non-prompt components of $X(3872)$ production cross sections, as well as their ratios to those of $\psi(2 S)$, may give further insight into the nature of this state and the mechanism of its production. The ATLAS experiment reported [13] differential cross sections for the production of $\psi(2 S)$ and $X(3872)$ states in the decay channel $J / \psi \pi^{+} \pi^{-}$, using $11.4 \mathrm{fb}^{-1}$ of $p p$ collisions at $\sqrt{s}=8 \mathrm{TeV}$. The production cross sections are measured in five bins of $J / \psi \pi^{+} \pi^{-}$ transverse momentum from 10 to $70 \mathrm{GeV}$ and each $p_{T}$ bin is further divided into intervals of pseudo-proper lifetime, to separate prompt and non prompt production (see Figure 4). As regards $\psi(2 S)$, the measured differential cross section times the product of the relevant branching fractions for prompt (non-prompt) production is well described by the NLO NRQCD (FONLL) model over the whole range of $p_{T}$. Also, the differential cross section for prompt production of $X(3872)$ is 

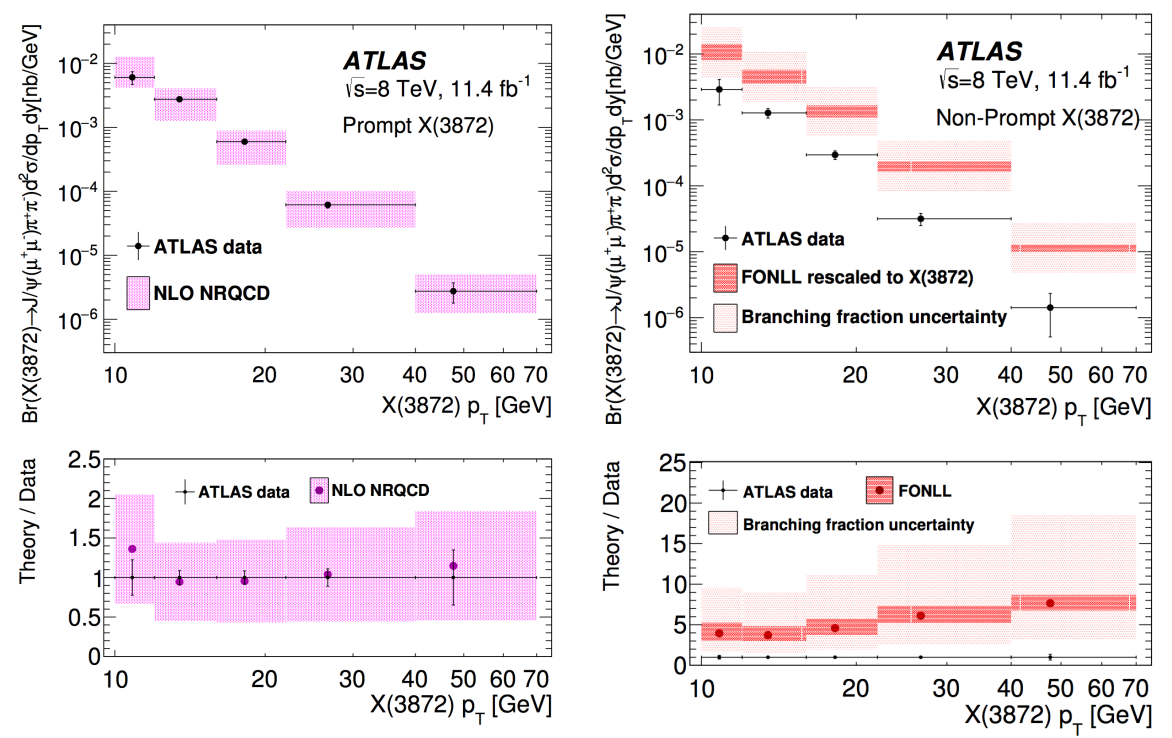

Figure 4: From [13]. Left: measured cross section times branching fractions as a function of $p_{T}$ for prompt $X(3872)$ compared to NLO NRQCD predictions with the $X(3872)$ modelled as a mixture of $\chi_{c 1}(2 P) D^{0} \bar{D}^{* 0}$ molecular state [14]. Right: measured cross section times branching fractions as a function of $p_{T}$ for nonprompt $X(3872)$ compared to the FONLL [15] model prediction, recalculated using the branching fraction estimate from [16]

described within theoretical uncertainty by the prediction of the NRQCD model which considers $X(3872)$ to be a mixture of a $\chi_{c 1}(2 P)$ and a $D^{0} \bar{D}^{* 0}$ state. On the other hand the prediction for $X(3872)$ non-prompt production, based on the FONLL model prediction for $\psi(2 S)$, overestimates the data by a factor 4 to 8 (see Figure 4).

Describing the non-prompt components of the signal distribution with a single effective lifetime $\tau_{e f f}$, it is measured that the signal from $X(3872)$ at low $p_{T}$ tends to have shorter lifetimes, possibly hinting at a different production mechanism at low $p_{T}$. An alternative model to explain this behaviour takes into account two non-prompt contributions with distinctly different effective lifetimes: a long-lived part, assumed to originate from $B^{ \pm}, B_{0}$, and $B_{s}$ mesons and from $b$-baryons; and a short-lived part, that would be due to the contribution of $B_{c}^{ \pm}$. The short-lived contributions to non-prompt $\psi(2 S)$ production are found to be not significant while the fraction of non-prompt $X(3872)$ from short-lived sources, integrated over the range $p_{T}>10 \mathrm{GeV}$ is measured to be $25 \pm 13$ (stat.) \pm 2 (syst.) \pm 5 (spin) \%. Since $B_{c}$ production is only a small fraction of the inclusive $b \bar{b}$ production, this value for the above ratio would imply that the production of $X(3872)$ in $B_{c}$ decays could be strongly enhanced compared to its production in the decays of other $b$-hadrons. 


\section{References}

[1] CMS Collaboration, Measurement of quarkonium production cross sections in pp collisions at $\sqrt{s}=13 \mathrm{TeV}$, Phys. Lett. B 780 (2018) 251 [arXiv:1710.11002v2 ]

[2] ATLAS Collaboration, Eur. Phys. J. C 78 (2018) 171

[3] H. Han et al., $\Upsilon(n S)$ and $\chi(n P)$ production at hadron colliders in nonrelativistic QCD., Phys. Rev. D 94, 014028 (2016). [arXiv:1410.8537]

[4] ATLAS Collaboration, Measurement of b-hadron pair production with the ATLAS detector in proton-proton collisions at $\sqrt{s}=8 \mathrm{TeV}$, JHEP 11 (2017) 062 [arXiv:1705.03374]

[5] CMS Collaboration, CMS PAS BPH-13-002 (2018) [https://cds.cern.ch/record/2621286/files/BPH-13-002-pas.pdf]

[6] QWG collaboration, Heavy quarkonium physics (2004) [arXiv:hep-ph/0412158], and references therein.

[7] J. Chang, C Wang and X.Wu, BCVEGPY2.0: An upgraded version of the generator BCVEGPY with the addition of hadroproduction of the $P$-wave $B_{c}$ states, Comput. Phys. Comm. 174 (2006) 11

[8] C.-F. Qiao, P. Sun, D. Yang, and R.-L. Zhu, Bc exclusive decays to charmonium and a light meson at next-to- leading-order accuracy, Phys. Rev. D 89034008 (2014).

[9] CMS Collaboration, Observation of the $\chi_{b 1}(3 P)$ and $\chi_{b 2}(3 P)$ and measurement of their masses, Phys. Rev. Lett. 121 (2018) 092002 [arXiv:1805.11192]

[10] CMS Collaboration, Measurement of the relative prompt production rate of $\chi_{c 2}$ and $\chi_{c 1}$ in $p p$ collisions at $\sqrt{s}=7 \mathrm{TeV}$, Eur. Phys. J. C 72 (2012) 2251, [arXiv:1210.0875]

[11] CMS Collaboration, Measurement of the production cross section ratio $\left(\chi_{b 2}(1 P)\right) / \sigma\left(\chi_{b 1}(1 P)\right)$ in pp collisions at $\sqrt{s}=8 \mathrm{TeV}$, Phys. Lett. B 743 (2015) 383 [arXiv:1409.5761.]

[12] Belle collaboration, Observation of a narrow charmonium-like state in exclusive $B^{\rightarrow} K^{+} \pi^{+} \pi^{-} J / \psi$ decays, Phys. Rev. Lett. 91 (2003) 262001 [hep-ex/0309032]

[13] ATLAS Collaboration, Measurements of $\psi(2 S)$ and $X(3872) \rightarrow J / \psi \pi^{+} \pi^{-}$production in pp collisions at $\sqrt{s}=8 \mathrm{TeV}$ with the ATLAS detector JHEP 01 (2017) 117 [arxiv:1610.09303]

[14] C. Meng, H. Han and K.T. Chao, X(3872) and its production at hadron colliders, arXiv:1304.6710

[15] M. Cacciari et al., Theoretical predictions for charm and bottom production at the LHC, JHEP 10 (2012) 137 [arXiv:1205.6344]

[16] P. Artoisenet and E. Braaten, Production of the X(3872) at the Tevatron and the LHC, Phys. Rev. D 81 (2010) 114018 [arXiv:0911.2016] . 